\title{
EDITORIAL
}

\section{Rebuilding a diseased lung: repair and regeneration}

\author{
Melanie Königshoff*, Sejal Saglani”, Benjamin J. Marsland ${ }^{\mp}$ and Oliver Eickelberg*
}

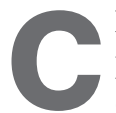
hronic lung diseases, including chronic obstructive pulmonary disease, asthma, lung cancer and pulmonary fibrosis, are the second leading cause of death in the world. Currently, only limited therapeutic options exist for chronic lung diseases; in particular, causative therapeutic approaches are missing. Lung transplantation remains the only available therapy for many patients with end-stage lung disease; however, the number of patients listed for lung transplantation by far surpasses the number of suitable donor organs.

Beyond lung transplantation, several approaches to repair and regenerate lung tissue, with the aim of restoring lung function, have made substantial progress in recent years and hold great promise for future therapies. Importantly, advancement in our understanding of the underlying pathogenesis of distinct chronic lung diseases will be crucial for the development of novel, targeted therapeutic approaches.

The mission of the European Respiratory Society (ERS) is to alleviate suffering from respiratory disease and promote lung health through research, knowledge sharing, and medical and public education. In support of this mission, the ERS hosts the annual Lung Science Conference (LSC) in Estoril, Portugal, which brings together leading experts (basic scientists, physician scientists and clinicians alike) on distinct topics of relevance to respiratory medicine. In addition, the ERS is strongly committed to encourage, nurture and mentor future generations of world class respiratory scientists and clinicians. The LSC represents one of the main platforms to encourage debate and interaction between young doctoral and postdoctoral scientists with established investigators. In particular, this is facilitated by special mentoring sessions and the Young Investigator Competition, which enables younger delegates to present themselves and their work to established investigators.

In April 2012, an outstanding line-up of international experts met on the occasion of the 10th LSC in Estoril, Portugal, to present and discuss novel findings and exchange novel ideas and opportunities that promote our ultimate aim of rebuilding a diseased lung. The meeting highlighted major novel findings in the fields of stem and progenitor cell functions, signalling

\footnotetext{
*Comprehensive Pneumology Center Munich (CPC-M), University Hospital of the LudwigMaximilians-University Munich and Helmholtz Zentrum München, German Center for Lung Research, Munich, Germany. ${ }^{\#}$ Respiratory Paediatrics, Royal Brompton Hospital, Imperial College London, London, UK. "Service de Pneumologie, Centre Hospitalier Universitaire Vaudois, Faculty of Biology and Medicine, University of Lausanne Vaudois Lausanne, Lausanne, Switzerland.

CORRESPONDENCE: O. Eickelberg, Comprehensive Pneumology Center Munich (CPC-M), University Hospital of the Ludwig-Maximilians-University Munich and Helmholtz Zentrum München Max-Lebsche-Platz 31, D-81377 Munich, Germany.E-mail: oliver.eickelberg@helmholtz-muenchen.de
}

systems, and tissue engineering. While most of the presentations from this LSC can be found on the ERS website (www.erseducation.org/pages/default.aspx?id2812), we will highlight some of the most important findings in the following paragraphs.

\section{CELLULAR PLASTICITY IN LUNG DEVELOPMENT AND DISEASE}

Normal lung development is controlled in a tight spatiotemporal fashion by several soluble factors, as well as cell types required for proper organ development [1]. It is likely that the capability of the lung to initiate repair and regeneration following any insult is altered over the life span of an organism. If and how the human adult lung regenerates are two of the most exciting questions that remain to be answered. Intriguingly, a recent report provides evidence that an adult human lung can regrow, as evidenced by an increased vital capacity, enlargement of the remaining left lung and increased alveolar numbers in a patient that underwent right-sided pneumonectomy more than 15 years ago [2]. These data raise hopes and expectations that deciphering the underlying processes will result in regenerative therapies for patients with end-stage lung disease.

In order to develop strategies to rebuild or regenerate the adult lung, we need to deepen our understanding of normal lung development and growth, and identify active developmental signalling systems that might present promising targets for therapeutic approaches. During his opening lecture, D. Warburton (University of Southern California, Los Angeles, CA, USA) outlined the general principles of lung branching and alveolar morphogenesis, highlighting that a number of pathways involved in alveolar and vascular development concomitantly guide lung growth [3]. Small changes in these processes may lead to prenatal or postnatal lung disease and, in combination with environmental exposures, predispose individuals to chronic lung disease in adulthood and ageing. One approach to rebuild lung function within a diseased lung is cell-based therapy. D. Warburton shared data from his laboratory showing that human amniotic fluid stem cells differentiate into epithelial lung lineages, thereby representing a promising source for cell-based therapies [4].

While human and mouse studies have been emerging, several key players involved in the processes of branching morphogenesis and epithelial homeostasis were not primarily identified in mice or humans, but evolved from studies in model systems, such as Xenopus or Drosophila. To gain deeper insight into airway development and maturation, C. Samakovlis (Stockholm University, Stockholm, Sweden) focused in his research presentation on the use of Drosophila as a model system [5]. He outlined that the mammalian homologue of 
Drosophila branchless, fibroblast growth factor (FGF)10, has been assigned a crucial role in mammalian lung development, as FGF10-/- mice embryos exhibit lung agenesis [6]. C. Samakovlis presented data on a tissue-specific, genome-wide RNAi screen that identified novel genetic and molecular networks involved in airway maturation, such as genes linked to endoplasmic reticulum-to-Golgi vesicle transport. These newly identified networks might be disturbed in chronic lung disease and modifications thereof could be useful for regenerative strategies.

\section{CIRCULATING AND RESIDENT PROGENITOR CELLS}

The bone marrow represents a potent source for several progenitor cell populations, such as haematopoietic stem cells, mesenchymal stem cells or fibrocytes. Many of these cell types have recently been reported to modify chronic lung disease; however, whether these cells may contribute directly to disease development or resolve lung injury is an ongoing debate. Following this line, S.M. Rankin (Imperial College London, London, UK) shared data demonstrating that bone marrowderived endothelial progenitor cells are recruited by a CXCL12/CXCR2 axis into the lung following allergen challenge, thereby influencing the angiogenic response, while not affecting lung inflammation [7]. This points to a critical role of CXCL12/CXCR2 for neovascularisation as observed in chronic lung diseases, such as asthma. The suitability of CXCL12/ CXCR2 inhibition as a novel therapeutic target is a subject for future studies. Next to bone marrow-derived progenitor cells, the lung itself represents a promising source of progenitor or stem cells. J. Rock (University of California San Francisco, San Francisco, CA, USA) highlighted in his presentation that our understanding of resident epithelial progenitor cell populations has made substantial advances in recent years. While several studies investigated conducting airway stem cell populations, the analysis of epithelial progenitor cells within the distal lung has been much less investigated [8]. J. Rock discussed recent findings, based on genetic lineage tracing, that unequivocally confirmed that alveolar epithelial type (AT)II cells differentiate into ATI cells in vivo. Interestingly, ATII cells expressing stromal markers were not detected upon lung injury [9]. These findings fuelled the recent debate over the source of the fibroblast pool in fibrotic lung disease. The transition of epithelial into mesenchymal cells (epithelial-tomesenchymal transition) was discussed as a significant source of the increased fibroblast pool, however; due to the highly dynamic nature of this process and the limited number of cellspecific markers, in particular in human tissue, this process warrants further investigation and confirmation.

The finding that resident stem cell niches exist within adult tissue was further corroborated by studies in other organs, such as the brain and the liver. M. Götz (Helmholtz Zentrum Munich, Munich, Germany) provided evidence that local progenitor cells exist within the adult brain and exhibit a high potential of plasticity in response to tissue injury, similar to stem cell populations in the lung [10]. The normal mature liver exhibits a high potential to regenerate upon chronic injury. S.J. Forbes (University of Edinburgh, Edinburgh, UK) highlighted recent data obtained from studying liver regeneration which revealed that the interplay between local tissue progenitor cells and macrophages tightly regulates cell differentiation [11].
The current concept underscores that macrophages, upon tissue injury and engulfment of cell debris, promote canonical Wnt signalling, thereby facilitating hepatocyte function and liver regeneration. Intriguingly, S.J. Forbes further outlined that bone marrow-derived macrophages are recruited to the liver during scar resolution and reduce liver fibrosis [12]. Clinical trials using autologous macrophage therapy have been initiated, the outcome of which might not only lead to novel therapies for liver fibrosis, but also lead to progress in cellbased therapies for organ fibrosis in general. Overall, these data strongly suggest that the processes involved in tissue repair and regeneration might be based, in part, on general mechanisms that could be targeted for therapy in several injured tissues.

\section{REGENERATIVE SIGNALLING MECHANISMS}

Recently, signalling pathways essential for organ development, such as Notch, Sonic Hedgehog, or Wnt signalling, have been demonstrated to be aberrantly altered in several chronic lung diseases. E.E. Morrisey (University of Pennsylvania, Philadelphia, PA, USA) focused on the effects of Wnt signalling during lung development. Based on the initial finding that specific Wnt ligands are essential for the earliest steps in lung bud formation, E.E. Morrisey pointed out that the Wnt ligands Wnt2 and 7b exhibit synergistic effects during lung development, in particular by promoting smooth muscle, as well as distal endoderm progenitor cell development, within the lung [13]. These data highlight that we need to more fully understand the spatio-temporal activity of distinct Wnt pathway components and their effect on specific cell types in chronically injured tissue in order to identify suitable therapeutic targets [14]. The importance of cell-specific crosstalk was further highlighted by delineation of the impairment of epithelial-mesenchymal crosstalk in the pathogenesis of idiopathic pulmonary fibrosis (IPF), as outlined by B. Crestani (Hôpital Xavier Bichat, INSERM, Paris, France). As one example, B. Crestani discussed the role of hepatocyte growth factor (HGF), which is an epithelial cell mitogen normally secreted by fibroblasts. In IPF, low levels of HGF have been found that may reduce the repair capacity of alveolar epithelial cells. Thus, increased HGF signalling may be an interesting target for future IPF therapies [15], but similar to other epithelial cell mitogens, it will be a major challenge to identify the level of signalling activity needed to stimulate repair, but not epithelial cell transformation and possibly carcinogenesis.

\section{TISSUE ENGINEERING AND LUNG TRANSPLANTATION}

Another promising approach for lung regeneration is based on tissue engineering. Here, the community has witnessed major breakthroughs in recent years. T.H. Petersen (Yale University, New Haven, CT, USA) shared data based on ex vivo engineered functional rat lungs, which were re-implanted into animals and retained proper lung function for a limited time, in vivo. This was achieved by using a decellularised lung acting as a scaffold, a bioreactor that resembled physiological conditions for cell expansion, and neonatal lung cells as an exogenous cellular source that repopulated the scaffold [16]. These data undoubtedly show great promise for future development of tissue engineered lungs; however, as pointed out by T.H. Petersen, the initial findings highlighted several questions that require extensive research, such as long-term organ function. 
Thus, lung transplantation still represents the only curative approach for patients with end-stage lung disease. In his presentation, A.J. Fisher (Newcastle University, Newcastle Upon Tyne, UK) highlighted that, although substantial advances in the field of lung transplantation have been made in recent years, the shortage of donor organs, immunosuppressive treatment, as well as long-term graft dysfunction and bronchiolitis obliterans syndrome, still present major hurdles that need to be overcome [17]. Recent progress has been made in ex vivo lung perfusion strategies that may lead to an increased number of donor organs suitable for transplantation and further allow application of novel cell-based therapies in combination with tissue engineering approaches [18].

Finally, during the 10th LSC, a unique meet-the-editor session was held. Four editors of major pulmonary journals (J.I. Sznajder of the American Journal of Respiratory and Critical Care Medicine, A.T. Dinh-Xuan of the European Respiratory Journal, K.B. Adler of the American Journal of Respiratory Cell and Molecular Biology, and S. Matalon of the American Journal of Physiology - Lung Cellular and Molecular Physiology) shared their thoughts and ideas on the "Present and future of publishing in lung science" with the audience. This session resulted in an active and long-lasting discussion and was very well received by all participants. A similar session will be included in the programme of the 11th LSC in 2013.

The focus of the 11th LSC in 2013 will be "Early origins and mechanisms of chronic lung disease." We invite you to join us and present and discuss recent advances within this exciting field from 15-17 March, 2013, in Estoril, Portugal.

\section{STATEMENT OF INTEREST}

Statements of interest for M. Königshoff, B.J. Marsland and O. Eickelberg can be found at www.erj.ersjournals.com/site/misc/statements.xhtml

\section{REFERENCES}

1 Cardoso WV, Lü J. Regulation of early lung morphogenesis: questions, facts and controversies. Development 2006; 133: $1611-1624$.
2 Butler JP, Loring SH, Patz S, et al. Evidence for adult lung growth in humans. N Engl J Med 2012; 367: 244-247.

3 Warburton D, El-Hashash A, Carraro G, et al. Lung organogenesis. Curr Top Dev Biol 2010; 90: 73-158.

4 Garcia O, Carraro G, Navarro S, et al. Cell-based therapies for lung disease. Br Med Bull 2012; 101: 147-161.

5 Tsarouhas V, Senti KA, Jayaram SA, et al. Sequential pulses of apical epithelial secretion and endocytosis drive airway maturation in Drosophila. Dev Cell 2007; 13: 214-225.

6 Min H, Danilenko DM, Scully SA, et al. Fgf-10 is required for both limb and lung development and exhibits striking functional similarity to Drosophila branchless. Genes Dev 1998; 12: 3156-3161.

7 Jones CP, Pitchford SC, Lloyd CM, et al. CXCR2 mediates the recruitment of endothelial progenitor cells during allergic airways remodeling. Stem Cells 2009; 27: 3074-3081.

8 Rock J, Konigshoff M. Endogenous lung regeneration: potential and limitations. Am J Respir Crit Care Med 2012; 189: 1213-1219.

9 Rock JR, Barkauskas CE, Cronce MJ, et al. Multiple stromal populations contribute to pulmonary fibrosis without evidence for epithelial to mesenchymal transition. Proc Natl Acad Sci USA 2011; 108: E1475-E1483.

10 Robel S, Berninger B, Götz M. The stem cell potential of glia: lessons from reactive gliosis. Nat Rev Neurosci 2011; 12: 88-104.

11 Boulter L, Govaere O, Bird TG, et al. Macrophage-derived Wnt opposes Notch signaling to specify hepatic progenitor cell fate in chronic liver disease. Nat Med 2012; 18: 572-579.

12 Thomas JA, Pope C, Wojtacha D, et al. Macrophage therapy for murine liver fibrosis recruits host effector cells improving fibrosis, regeneration, and function. Hepatology 2011; 53: 2003-2015.

13 Miller MF, Cohen ED, Baggs JE, et al. Wnt ligands signal in a cooperative manner to promote foregut organogenesis. Proc Natl Acad Sci USA 2012; 109: 15348-15353.

14 Beers MF, Morrisey EE. The three R's of lung health and disease: repair, remodeling, and regeneration. J Clin Invest 2011; 121: 2065-2073.

15 Crestani B, Marchand-Adam S, Quesnel C, et al. Hepatocyte growth factor and lung fibrosis. Proc Am Thora Soc 2012; 9: 158-163.

16 Petersen TH, Calle EA, Zhao L, et al. Tissue-engineered lungs for in vivo implantation. Science 2010; 329: 538-541.

17 Mahida RY, Wiscombe S, Fisher AJ. Current status of lung transplantation. Chron Respir Dis 2012; 9: 131-145.

18 Sanchez PG, Bittle GJ, Burdorf L, et al. State of art: clinical ex vivo lung perfusion: rationale, current status, and future directions. J Heart Lung Transplant 2012; 31: 339-348. 